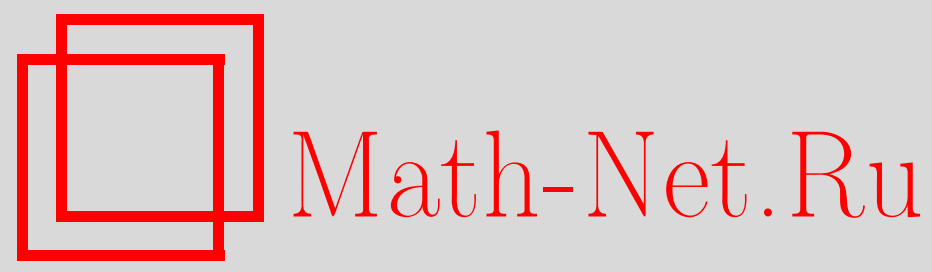

М. А. Подколзина, О полноте и $A$-полноте $S$-множеств детерминированных функций, содержащих все одноместные детерминированные $S$-функции, Дискрет. матем., 2009, том 21, выпуск 2, 75-87

DOI: https://doi.org/10.4213/dm1047

Использование Общероссийского математического портала Math-Net.Ru подразумевает, что вы прочитали и согласны с пользовательским соглашением http://www . mathnet.ru/rus/agreement

Параметры загрузки:

IP : 54.209 .52 .79

26 апреля 2023 г., 11:46:53 


\title{
О полноте и $A$-полноте $S$-множеств детерминированных функций, содержащих все одноместные детерминированные $S$-функции
}

\author{
() 2009 г. М. А. Подколзина
}

\begin{abstract}
Исследуется задача о полноте систем, состоящих из $S$-функций, то есть таких детерминированных функций, что в каждом состоянии вычисляющего их автомата реализуются функции, не выпускающие ни одного значения. Предполагается, что каждой рассматриваемой на полноту системе $S$-функций принадлежат все $S$-функции, зависящие не более, чем от одной переменной. В терминах сохранения отношений описаны все $A$-предполные классы для таких систем. Показано, что существует алгоритм для распознавания $A$-полноты $S$-систем одноместных детерминированных функций, содержащих все одноместные детерминированные $S$-функции.
\end{abstract}

Пусть $k \geqslant 2, \tau \geqslant 1$ и $P_{k}^{\tau}-$ функциональная система, элементами которой являются детерминированные функции, определенные на словах длины $\tau$, составленных из букв алфавита $E_{k}=\{0,1, \ldots, k-1\}$, а операциями - операции суперпозиции. Заметим, что любая детерминированная функция из $P_{k}^{\tau}$ может быть вычислена некоторым конечным автоматом за первые $\tau$ тактов его работы. Пусть $\mathfrak{M} \subseteq P_{k}^{\tau}$. Замыкание множества $\mathfrak{M}$ относительно операций суперпозиции обозначим через [M]. Множество $\mathfrak{M} \subseteq P_{k}^{\tau}$ называется замкнутым, если оно совпадает со своим замыканием.

Определение 1. Пусть $k \geqslant 2, \tau \geqslant 1, \mathfrak{M} \subseteq P_{k}^{\tau}$. Множество $\mathfrak{M}$ называется полным в $P_{k}^{\tau}$, если $[\mathfrak{M}]=P_{k}^{\tau}$.

Известно $[1,5]$, что для любых $k \geqslant 2, \tau \geqslant 1$ существуют конечные системы, полные в $P_{k}^{\tau}$.

Из определения детерминированных функций $[1,3]$ следует, что проблема полноты в $k$-значных логиках эквивалентна проблеме полноты в $P_{k}^{1}$, то есть при $\tau=1$. Вместе с тем, при $\tau \geqslant 2$ существует принципиальное различие между функциональной системой, элементами которой являются функции $k$-значных логик, и функциональной системой $P_{k}^{\tau}$. Множество всех детерминированных отображений, рассматриваемых на словах длины $\tau$, порождает специальное замкнутое подмножество в $k^{\tau}$-значных логиках, существенно зависящее от двух параметров, параметра $k$ и параметра $\tau[1,4]$.

Определение 2. Пусть $k \geqslant 2, \tau \geqslant 1$. Замкнутое множество $\mathfrak{\Re} \subset P_{k}^{\tau}$ называется предполным классом в $P_{k}^{\tau}$, если $\mathfrak{N}$ не является полным в $P_{k}^{\tau}$, но для любой детерминированной функции $f \notin \mathfrak{N}$ замыкание множества $\mathfrak{N} \cup\{f\}$ совпадает с $P_{k}^{\tau}$. 
Используя естественную аналогию между проблемой полноты в $P_{k}^{\tau}$ и проблемой полноты в конечнопорожденных замкнутых классах $k^{\tau}$-значной логики, можно показать, что всякое множество является полным в $P_{k}^{\tau}$ тогда и только тогда, когда оно целиком не содержится ни в одном из предполных в $P_{k}^{\tau}$ классов; совокупность предполных в $P_{k}^{\tau}$ классов конечна, может быть описана эффективно и образует минимальную критериальную систему для распознавания полноты систем функций из $P_{k}^{\tau}$; при этом множество предполных классов в $P_{k}$ изоморфно некоторому подмножеству предполных классов в $P_{k}^{\tau}$.

В общем случае для любых $k \geqslant 2, \tau \geqslant 1$ задача о полноте в $P_{k}^{\tau}$ решена в [1]. В терминах сохранения отношений описаны все предполные в $P_{k}^{\tau}$ классы. Однако это описание оказалось довольно сложным. В связи с этим представлялась естественной задача об исследовании на полноту систем детерминированных функций, которые обладают некоторыми наперед заданными и вместе с тем достаточно общими свойствами $[1,3]$.

Определение 3. Пусть $k \geqslant 2, \tau \geqslant 1$ и $f\left(x_{1}, \ldots, x_{n}\right) \in P_{k}^{\tau}$. Детерминированная функция $f\left(x_{1}, \ldots, x_{n}\right)$ называется $S$-функцией, если в любом состоянии вычисляющего ее автомата реализуется функция $k$-значной логики, не выпускающая ни одного значения из множества $E_{k}$.

Определение 4. Пусть $\mathfrak{M} \subset P_{k}^{\tau}$. Множество $\mathfrak{M}$ называется $S$-множеством, если любая функция из $\mathfrak{M}$ является $S$-функцией.

Множество всех $S$-функций, принадлежащих $P_{k}^{\tau}$, обозначим $S\left(P_{k}^{\tau}\right)$.

Определение 5. Пусть $k \geqslant 2, \tau \geqslant 1 ; S$-множество $\mathfrak{N} \subset P_{k}^{\tau}$ называется $S$-предполным классом в $P_{k}^{\tau}$, если $\mathfrak{N}$ не является полным в $P_{k}^{\tau}$, но для любой детерминированной $S$-функции $f \notin \Re$ замыкание множества $\mathfrak{N} \cup\{f\}$ совпадает с $P_{k}^{\tau}$.

В $[1,3]$ доказана следующая теорема.

Теорема 1. Пусть $k \geqslant 2, \tau \geqslant 1 ; S$-множество $\mathfrak{M} \subset P_{k}^{\tau}$ является полным в $P_{k}^{\tau}$ тогда и только тогда, когда $\mathfrak{M}$ не принадлежит иеликом ни одному из $S$-предполных в $P_{k}^{\tau}$ классов, причем число $S$-предполных классов в $P_{k}^{\tau}$ конечно.

Так же, как и в общем случае, совокупность всех $S$-предполных классов в $P_{k}^{\tau}$ описывается с использованием аппарата сохранения отношений.

Определение 6. Пусть $k \geqslant 2, \tau \geqslant 1$. Пусть $h \geqslant 1, T=\left(t_{1}, \ldots, t_{h}\right)$ - произвольный набор положительных целых чисел такой, что $\max \left\{t_{1}, \ldots, t_{h}\right\} \leqslant \tau$. Пусть $E_{k}^{T}=E_{k}^{t_{1}} \times \ldots \times E_{k}^{t_{h}}$, где для любого $i, 1 \leqslant i \leqslant h, E_{k}^{t_{i}}-$ множество всех слов длины $t_{i}$, составленных из букв алфавита $E_{k}$. Произвольное непустое множество $R \subseteq E_{k}^{T}$ называется отношением, заданным на $E_{k}^{T}$, а число $h-$ арностью этого отношения.

Определение 7. Пусть $k \geqslant 2, \tau \geqslant 1$. Детерминированная функция $f\left(x_{1}, \ldots, x_{n}\right)$ из $P_{k}^{\tau}$ сохраняет отношение $R \subset E_{k}^{T}$, если для любой совокупности $\left(a_{1}^{1}, \ldots, a_{h}^{1}\right), \ldots,\left(a_{1}^{n}, \ldots, a_{h}^{n}\right)$ элементов из $R$ набор $\left(f\left(a_{1}^{1}, \ldots, a_{1}^{n}\right), \ldots, f\left(a_{h}^{1}, \ldots, a_{h}^{n}\right)\right)$ также принадлежит $R$. Множество $\mathfrak{M} \subseteq P_{k}^{\tau}$ сохраняет отношение $R$, если каждая функция из $\mathfrak{M}$ сохраняет это отношение $[1,3,9]$.

Множество всех детерминированных функций, сохраняющих некоторое отношение $R$, обозначим через $U(R)$, а множество всех $S$-функций, сохраняющих $R$, - через $S(U(R))$. Нетрудно видеть, что для любого $R$ множество $U(R)$ замкнуто, а множество $S(U(R))$, вообще говоря, не является таковым. 
В данной работе для любых $k \geqslant 2, \tau \geqslant 1$ рассматривается задача, аналогичная классической задаче Слупецкого-Яблонского $[4,7]$ для $k$-значных логик. Пусть $S\left(P_{k}^{\tau}(1)\right)-$ множество всех $S$-функций из $P_{k}^{\tau}$, существенно зависящих только от одной переменной. Пусть $M_{k}^{\tau}-$ совокупность всех $S$-множеств $\mathfrak{M} \subset P_{k}^{\tau}$ таких, что $S\left(P_{k}^{\tau}(1)\right) \subseteq \mathfrak{M}$. Возникает вопрос, какой должна быть минимальная критериальная система для распознавания полноты $S$-множеств, принадлежащих $M_{k}^{\tau}$. Очевидно, эта критериальная система состоит из всех тех и только тех $S$-предполных классов, которые содержат все одноместные $S$-функции из $P_{k}^{\tau}$. Известно, что при $\tau=1$, то есть в $k$-значных логиках, существует единственный предполный класс, содержащий все функции $k$-значной логики, зависящие не более, чем от одной переменной. Заметим также, что в общем случае при рассмотрении произвольных подмножеств $P_{k}^{\tau}$ данная задача решена в [6].

Как было отмечено выше, множество всех $S$-предполных классов в терминах сохранения отношений описано в [3]. Совокупность всех отношений $R$ таких, что множество $S(U(R))$ совпадает с $S$-предполным классом в $P_{k}^{\tau}$, разбивается на шесть семейств $Z(k, \tau)$, $D(k, \tau), N(k, \tau), V(k, \tau), L(k, \tau)$ и $I(k, \tau)$.

Опишем эти семейства.

Семейство $Z(k, \tau) . Z(k, \tau) \neq \varnothing$ для любых $k \geqslant 2, \tau \geqslant 1$.

Унарное отношение $R$ принадлежит семейству $Z(k, \tau)$ тогда и только тогда, когда $R \subset E_{k}^{t}$ для некоторого $t \leqslant \tau$, причем при $\tau \geqslant 2, t \geqslant 2$ имеет место следующее. Для любого $a \in E_{k}^{t-1}, a=(a(1), \ldots, a(t-1))$ существуют $a(t) \in E_{k}, a^{\prime}(t) \in E_{k}$ такие, что $a(t) \neq a^{\prime}(t),(a(1), \ldots, a(t-1), a(t))$ принадлежит, а $\left(a(1), \ldots, a(t-1), a^{\prime}(t)\right)$ не принадлежит $R$.

Семейство $D(k, \tau) . D(k, \tau) \neq \varnothing$ для любых $k \geqslant 3, \tau \geqslant 1$.

Бинарное отношение $R$ принадлежит семейству $D(k, \tau)$ тогда и только тогда, когда $R \subset E_{k}^{t} \times E_{k}^{t}$ для некоторого $t, 1 \leqslant t \leqslant \tau, R$ является определенным на $E_{k}^{t}$ отношением эквивалентности, причем имеет место следующее. Существует принадлежащий $R$ набор $\left(a_{1}, a_{2}\right)$ такой, что $a_{1}(1)=a_{2}(1), \ldots, a_{1}(t-1)=a_{2}(t-1)$. Кроме того, для любого $a \in E_{k}^{t}$ существует $a^{\prime} \in E_{k}^{t}$ такой, что $a(t) \neq a^{\prime}(t)$, набор $\left(a, a^{\prime}\right)$ не принадлежит $R$ и $a(1)=a^{\prime}(1), \ldots, a(t-1)=a^{\prime}(t-1)$ при $\tau \geqslant 2, t \geqslant 2$.

Семейство $N(k, \tau) . N(k, \tau) \neq \varnothing$ для любых $k \geqslant 2, \tau \geqslant 1$.

Бинарное отношение $R$ принадлежит семейству $N(k, \tau)$ тогда и только тогда, когда $R \subset E_{k}^{t} \times E_{k}^{t}$ для некоторого $t, 1 \leqslant t \leqslant \tau$, существует определенная на $E_{k}^{t}$ подстановка $\sigma_{R}$, которая разлагается в произведение циклов одинаковой простой длины $p \geqslant 2$ и график которой совпадает с $R$, причем, если $\tau \geqslant 2, t \geqslant 2$ и набор $\left(a_{1}, a_{2}\right)$ принадлежит $R$, то $a_{1}(1)=a_{2}(1), \ldots, a_{1}(t-1)=a_{2}(t-1)$. Таким образом, $\left(a, \sigma_{R}(a)\right) \in R$ для любого $a \in E_{k}^{t}$ и $\left(a_{2}, \sigma_{R}\left(a_{1}\right)\right.$ для любого набора $\left(a_{1}, a_{2}\right)$ из $R$.

Пусть $k \geqslant 2, \tau \geqslant 1$. Пусть $\Sigma-$ множество всех подстановок (перестановок), определенных на $E_{k}$. Пусть $t \in\{1, \ldots, \tau\}$ и $\Phi_{t}-$ совокупность отображений множества $E_{k}^{t}$ в $\Sigma$ такая, что $\varphi(a)=\varphi\left(a^{\prime}\right)$ при $t=1$ для любых $\varphi \in \Phi_{t}, a \in E_{k}, a^{\prime} \in E_{k}$, а при $\tau \geqslant 2$, $t \geqslant 2$ справедливо равенство $\varphi(a)=\varphi\left(a^{\prime}\right)$ для любых $\varphi \in \Phi_{t}, a \in E_{k}^{t}, a^{\prime} \in E_{k}^{t}$, если $a(1)=a^{\prime}(1), \ldots, a(t-1)=a^{\prime}(t-1)$. Подстановку, которую отображение $\varphi \in \Phi_{t}$ ставит в соответствие элементу $a \in E_{k}^{t}$, обозначим через $\sigma_{\varphi(a)}$.

Семейство $V(k, \tau) . V(k, \tau) \neq \varnothing$ для любых $k \geqslant 2, \tau \geqslant 2$.

Бинарное отношение $R$ принадлежит семейству $V(k, \tau)$, если для некоторых $t \leqslant \tau$, $\varphi \in \Phi_{t}$ имеет место следующее: $R \subset E_{k}^{t} \times E_{k}^{t}$, набор $\left(a_{1}, a_{2}\right)$ принадлежит $R$ тогда и 
только тогда, когда либо $a_{1}(t-1)=a_{2}(t-1), a_{1}(t)=a_{2}(t)$, либо $a_{1}(t-1) \neq a_{2}(t-1)$ и существует $\alpha \in E_{k}$ такое, что $a_{1}(t)=\sigma_{\varphi\left(a_{1}\right)}(\alpha), a_{2}(t)=\sigma_{\varphi\left(a_{2}\right)}(\alpha)$, причем $a_{1}(1)=a_{2}(1), \ldots, a_{1}(t-2)=a_{2}(t-2)$ при $\tau \geqslant 3, t \geqslant 3$.

Пусть $k=p^{m}$, где $p-$ простое число, $p \geqslant 2, m \geqslant 1$. Пусть $G=\left\langle E_{k}, \oplus\right\rangle-$ произвольная элементарная абелева $p$-группа. В каждой такой группе всякий ненулевой элемент имеет порядок $p$. Пусть $\tau \geqslant 1$.

Семейство $L(k, \tau) . L(k, \tau) \neq \varnothing$ для любого $\tau \geqslant 1$, если $k=p^{m}$, где $p-$ простое число, $p \geqslant 2, m \geqslant 1$.

Отношение $R$, арность которого равна 4 , принадлежит семейству $L(k, \tau)$, если для некоторых $t, 1 \leqslant t \leqslant \tau, \varphi \in \Phi_{t}$, справедливо включение $R \subset E_{k}^{t} \times E_{k}^{t} \times E_{k}^{t} \times E_{k}^{t}$ и набор $\left(a_{1}, a_{2}, a_{3}, a_{4}\right)$ принадлежит $R$ тогда и только тогда, когда

$$
\sigma_{\varphi\left(a_{1}\right)}\left(a_{1}(t)\right) \oplus \sigma_{\varphi\left(a_{1}\right)}\left(a_{2}(t)\right)=\sigma_{\varphi\left(a_{1}\right)}\left(a_{3}(t)\right) \oplus \sigma_{\varphi\left(a_{1}\right)}\left(a_{4}(t)\right),
$$

причем

$$
a_{1}(1)=a_{2}(1)=a_{3}(1)=a_{4}(1), \ldots, a_{1}(t-1)=a_{2}(t-1)=a_{3}(t-1)=a_{4}(t-1)
$$

при $\tau \geqslant 2, t \geqslant 2$.

Определенное в [1] семейство $I(k, \tau)$ разобьем на два подсемейства, семейство $I_{0}(k, \tau)$ и семейство $I_{1}(k, \tau)$.

Семейство $I_{0}(k, \tau)$. $I_{0}(k, \tau \neq \varnothing$ для всякого $\tau \geqslant 1$ и любого $k>5$, представимого в виде $k=h^{m}$, где $h \geqslant 5, m>1$.

Пусть $h \geqslant 5, m>1, k=h^{m}$. Бинарное отношение $R$ принадлежит подсемейству $I_{h}(k, \tau)$ семейства отношений $I_{0}(k, \tau)$, если для некоторых $t, 1 \leqslant t \leqslant \tau$, $\varphi \in \Phi_{t}$, справедливо включение $R \subset E_{k}^{t} \times E_{k}^{t}$, набор $\left(a_{1}, a_{2}\right)$ принадлежит $R$ тогда и только тогда, когда для любого $i, 1 \leqslant i \leqslant m, i$-е компоненты чисел $\sigma_{\varphi\left(a_{1}\right)}\left(a_{1}(t)\right), \sigma_{\varphi\left(a_{1}\right)}\left(a_{2}(t)\right)$ при разложении их по степеням $h$ различны и $a_{1}(1)=a_{2}(1), \ldots, a_{1}(t-1)=a_{2}(t-1)$ при $\tau \geqslant 2, t \geqslant 2$. Семейство отношений $I_{0}(k, \tau)$ есть объединение семейств $I_{h}(k, \tau)$, взятое по всем $h \geqslant 5$ таким, что $h^{m}=k$, где $m>1$.

Семейство $I_{1}(k, \tau) . I_{1}(k, \tau) \neq \varnothing$ для любых $k \geqslant 5, \tau \geqslant 1$.

Бинарное отношение $R$ принадлежит семейству $I_{1}(k, \tau)$, если для некоторого $t$, $1 \leqslant t \leqslant \tau$, имеет место следующее: $R \subset E_{k}^{t} \times E_{k}^{t}$, набор $\left(a_{1}, a_{2}\right)$ принадлежит $R$ тогда и только тогда, когда $a_{1}(t) \neq a_{2}(t)$, причем $a_{1}(1)=a_{2}(1), \ldots, a_{1}(t-1)=a_{2}(t-1)$ при $\tau \geqslant 2, t \geqslant 2$.

Пусть $k \geqslant 2, \tau \geqslant 1, W(k, \tau)$ - объединение семейств $Z(k, \tau), D(k, \tau), N(k, \tau), V(k, \tau)$, $L(k, \tau), I_{0}(k, \tau), I_{1}(k, \tau)$.

Имеют место следующие утверждения [1].

Теорема 2. Пусть $k \geqslant 2, \tau \geqslant 1$. Произвольное $S$-множество $\mathfrak{M} \subset P_{k}^{\tau}$ является полньмм в $P_{k}^{\tau}$ тогда и только тогда, когда $\mathfrak{M}$ не сохраняет ни одного отношения $R$ из $W(k, \tau)$.

Теорема 3. Пусть $k \geqslant 2, \tau \geqslant 1$. Пусть $\mathfrak{N}-$ произвольныий $S$-предполный класс из $P_{k}^{\tau}$. Тогда существует отношение $R \in W(k, \tau)$ такое, что $\mathfrak{\Re}=S(U(R))$. 
Теорема 4. Пусть $k \geqslant 2, \tau \geqslant 1$. Пусть $R \in W(k, \tau)$. Тогда множество $S(U(R))$ образует $S$-предполный класс в $P_{k}^{\tau}$.

Определение 8. Пусть $h \geqslant 1, T=\left(t_{1}, \ldots, t_{h}\right)$, причем $\max \left\{t_{1}, \ldots, t_{h}\right\} \leqslant \tau$. Пусть $R \subset E_{k}^{T}$. Отношение $R$ назовем $C S$-отношением, если для всякого набора $\left(a_{1}, \ldots, a_{h}\right)$ из $R$ и для любой $S$-функции $f(x) \in S\left(P_{k}^{\tau}(1)\right)$ набор $\left(f\left(a_{1}\right), \ldots, f\left(a_{h}\right)\right)$ также принадлежит $R$.

Очевидно, что справедлива следующая теорема.

Теорема 5. Пусть $k \geqslant 2, \tau \geqslant 1$. Произвольное $S$-множество $\mathfrak{M}$, принадлежашее совокупности $S$-множеств $M_{k}^{\tau}$, является полным в $P_{k}^{\tau}$ тогда и только тогда, когда $\mathfrak{M}$ не сохраняет ни одного CS-отношения $R$ из $W(k, \tau)$.

Целью данной работы является описание всех $C S$-отношений, принадлежащих множеству $W(k, \tau)$.

Для любого $\tau \geqslant 1$ рассмотрим три семейства отношений, семейства $L_{2}(2, \tau), L_{3}(3, \tau)$ и $L_{4}(4, \tau)$.

Семейство $L_{2}(2, \tau)$. $L_{2}(2, \tau) \neq \varnothing$ для любого $\tau \geqslant 1$.

Отношение $R$, арность которого равна 4 , принадлежит семейству $L_{2}(2, \tau)$, если для некоторого $t, 1 \leqslant t \leqslant \tau$, имеет место следующее: $R \subset E_{2}^{t} \times E_{2}^{t} \times E_{2}^{t} \times E_{2}^{t}$, набор $\left(a_{1}, a_{2}, a_{3}, a_{4}\right)$ принадлежит $R$ тогда и только тогда, когда справедливо сравнение $a_{1}(t) \oplus a_{2}(t)(\bmod 2)=a_{3}(t) \oplus a_{4}(t)(\bmod 2)$, причем при $\tau \geqslant 2, t \geqslant 2$

$$
a_{1}(1)=a_{2}(1)=a_{3}(1)=a_{4}(1), \ldots, a_{1}(t-1)=a_{2}(t-1)=a_{3}(t-1)=a_{4}(t-1) .
$$

Семейство $L_{3}(3, \tau)$. $\left(L_{3}(3, \tau) \neq \varnothing\right.$ при любом $\tau \geqslant 1$.

Отношение $R$, арность которого равна 4 , принадлежит семейству $L_{3}(3, \tau)$, если для некоторого $t, 1 \leqslant t \leqslant \tau$, имеет место следующее: $R \subset E_{3}^{t} \times E_{3}^{t} \times E_{3}^{t} \times E_{3}^{t}$, набор $\left(a_{1}, a_{2}, a_{3}, a_{4}\right)$ принадлежит $R$ тогда и только тогда, когда справедливо сравнение $a_{1}(t) \oplus a_{2}(t)(\bmod 3)=a_{3}(t) \oplus a_{4}(t)(\bmod 3)$, причем при $\tau \geqslant 2$ и $t \geqslant 2$

$$
a_{1}(1)=a_{2}(1)=a_{3}(1)=a_{4}(1), \ldots, a_{1}(t-1)=a_{2}(t-1)=a_{3}(t-1)=a_{4}(t-1) .
$$

Семейство $L_{4}(4, \tau) .\left(L_{4}(4, \tau) \neq \varnothing\right.$ для любого $\tau \geqslant 1$.

Отношение $R$, арность которого равна 4 , принадлежит семейству $L_{4}(4, \tau)$, если для некоторого $t, 1 \leqslant t \leqslant \tau$, имеет место следующее: $R \subset E_{4}^{t} \times E_{4}^{t} \times E_{4}^{t} \times E_{4}^{t}$, набор $\left(a_{1}, a_{2}, a_{3}, a_{4}\right)$ принадлежит $R$ тогда и только тогда, когда справедливо равенство $a_{1}(t)+a_{2}(t)=a_{3}(t)+a_{4}(t)$, где коммутативная операция + на множестве $E_{4}$ определяется равенствами $0+0=0,0+1=1,0+2=2,0+3=3,1+1=0$, $1+2=3,1+3=2,2+2=0,2+3=1,3+3=0$. При этом, если $\tau \geqslant 2, t \geqslant 2$, то

$$
a_{1}(1)=a_{2}(1)=a_{3}(1)=a_{4}(1), \ldots, a_{1}(t-1)=a_{2}(t-1)=a_{3}(t-1)=a_{4}(t-1) .
$$

Очевидно, семейства отношений $L_{2}(2, \tau), L_{3}(3, \tau)$ и $L_{4}(4, \tau)$ являются подсемействами семейств отношений $L(2, \tau), L(3, \tau)$ и $(L 4, \tau)$ соответственно. 
Теорема 6. Справедливы следующие утверждения.

(1) Пусть $\tau \geqslant 1, k=2$. Отношение $R$ из $W(2, \tau)$ является CS-отношением тогда и только тогда, когда $R \in N(2, \tau) \cup L_{2}(2, \tau)$.

(2) Пусть $\tau \geqslant 1, k=3$. Отношение $R$ из $W(3, \tau)$ является CS-отношением тогда $и$ только тогда, когда $R \in L_{3}(3, \tau)$.

(3) Пусть $\tau \geqslant 1, k=4$. Отношение $R$ из $W(4, \tau)$ является CS-отношением тогда $и$ только тогда, когда $R \in L_{4}(4, \tau)$.

(4) Пусть $\tau \geqslant 1, k \geqslant 5$. Отношение $R$ из $W(k, \tau)$ является CS-отношением тогда $и$ только тогда, когда $R \in I_{1}(k, \tau)$.

Справедливость утверждений теоремы 6 будет следовать из справедливости приведенных ниже предложений 1-11.

Предложение 1. Пусть $k \geqslant 2, \tau \geqslant 1, R-$ произвольное отношение из семейства $Z(k, \tau)$. Тогда $S\left(P_{k}^{\tau}(1)\right) \not \subset S(U(R))$.

Доказательство. По определению семейства отношений $Z(k, \tau)$, найдется $t, 1 \leqslant t \leqslant \tau$, такое, что $R \subset E_{k}^{t}$. Пусть $a \in R, a^{\prime} \in E_{k}^{t}$, но $a^{\prime} \notin R$. Очевидно, существует $S$-функция $f(x)$ такая, что $f(a)=a^{\prime}$. Отсюда следует, что $f(x) \notin S(U(R))$ и $S\left(P_{k}^{\tau}(1)\right) \not \subset S(U(R))$.

Предложение 2. Пусть $k \geqslant 3, \tau \geqslant 1, R$ - произвольное отношение из семейства $D(k, \tau)$. Тогда $S\left(P_{k}^{\tau}(1)\right) \not \subset S(U(R))$.

Доказательство. По определению семейства отношений $D(k, \tau)$, найдется $t, 1 \leqslant t \leqslant \tau$, такое, что $R \subset E_{k}^{t} \times E_{k}^{t}$. Кроме того, существуют $a \in E_{k}^{t}, a_{1} \in E_{k}^{t}, a_{2} \in E_{k}^{t}$ такие, что набор $\left(a, a_{1}\right)$ принадлежит, а набор $\left(a, a_{2}\right)$ не принадлежит отношению $R$. При этом, если $\tau \geqslant 2, t \geqslant 2$, то $a_{1}(1)=a_{2}(1)=a(1), \ldots, a_{1}(t-1)=a_{2}(t-1)=a(t-1)$. Нетрудно видеть, что существует $S$-функция $f(x)$ такая, что $f(a)=a, f\left(a_{1}\right)=a_{2}$. Отсюда следует, что $f(x) \notin S(U(R))$ и $S\left(P_{k}^{\tau}(1)\right) \not \subset S(U(R))$.

Предложение 3. Пусть $k \geqslant 3, \tau \geqslant 1, R$ - произвольное отношение из семейства $N(k, \tau)$. Тогда $S\left(P_{k}^{\tau}(1)\right) \not \subset S(U(R))$.

Доказательство. Пусть для некоторого $t, 1 \leqslant t \leqslant \tau, R \subset E_{k}^{t} \times E_{k}^{t}$, набор $\left(a_{1}, a_{2}\right)$ принадлежит $R$. Так как $k \geqslant 3$, из определения семейства отношений $N(k, \tau)$ следует, что существует $a_{2}^{1} \in E_{k}^{t}$ такое, что $a_{2}^{1} \neq a_{1}, a_{2}^{1} \neq a_{2}$, набор $\left(a_{1}, a_{2}^{1}\right)$ не принадлежит $R$ и, если $\tau \geqslant 2, t \geqslant 2$, то $a_{2}^{\prime}(1)=a_{2}(1)=a_{1}(1), \ldots, a_{2}^{\prime}(t-1)=a_{2}(t-1)=a_{1}(t-1)$. Вместе с тем, как легко видеть, существует $S$-функция $f(x)$ такая, что $f\left(a_{1}\right)=a_{1}, f\left(a_{2}\right)=a_{2}^{1}$. Очевидно, $f(x) \notin S(U(R))$ и $S\left(P_{k}^{\tau}(1)\right) \not \subset S(U(R))$.

Предложение 4. Пусть $k=2, \tau \geqslant 1, R$ - произвольное отношение из семейства $N(2, \tau)$. Тогда $S\left(P_{2}^{\tau}(1)\right) \subset S(U(R))$.

Доказательство. Пусть для некоторого $t, 1 \leqslant t \leqslant \tau, R \subset E_{k}^{t} \times E_{k}^{t}$. Из определения семейства отношений $N(2, \tau)$ следует, что набор $\left(a_{1}, a_{2}\right)$ принадлежит $R$ лишь в том случае, когда $a_{2}(t)=\bar{a}_{1}(t)$ и $a_{2}(1)=a_{1}(1), \ldots, a_{2}(t-1)=a_{1}(t-1)$ при $\tau \geqslant 2, t \geqslant 2$. Пусть $f(x)$ - произвольная $S$-функция из $P_{2}^{\tau}$. Пусть $f\left(a_{1}\right)=a_{1}^{\prime}, f\left(a_{2}\right)=a_{2}^{\prime}$. Очевидно, $a_{2}^{\prime}(t)=\bar{a}_{1}(t)$ и при $\tau \geqslant 2, t \geqslant 2, a_{2}^{\prime}(1)=a_{2}(1), \ldots, a_{2}^{\prime}(t-1)=a_{2}(t-1)$. Таким образом, $f(x) \in s(U(R))$ и $S\left(P_{2}^{\tau}(1)\right) \subset S(U(R))$. 
Предложение 5. Пусть $k \geqslant 2, \tau \geqslant 2, R-$ произвольное отночение из семейства $V(k, \tau)$. Тогда $S\left(P_{k}^{\tau}(1)\right) \not \subset S(U(R))$.

Доказательство. Пусть для некоторых $t, 2 \leqslant t \leqslant \tau, \varphi \in \Phi_{t}, R \subset E_{k}^{t} \times E_{k}^{t}$, набор $\left(a_{1}, a_{2}\right)$ принадлежит $R$, причем $a_{1}(t-1) \neq a_{2}(t-1)$ и если $\tau \geqslant 3, t \geqslant 3$, то $a_{2}(1)=a_{1}(1), \ldots, a_{2}(t-2)=a_{1}(t-2)$. Не ограничивая общности, будем считать, что $a_{1}(t)=\sigma_{\varphi\left(a_{1}\right)}(0), a_{2}(t)=\sigma_{\varphi\left(a_{2}\right)}(0)$. Пусть $\left(a_{1}, a_{2}^{\prime}\right)-$ элемент из $E_{k}^{t} \times E_{k}^{t}$ такой, что $a_{2}^{\prime}(1)=a_{2}(1), \ldots, a_{2}^{\prime}(t-2)=a_{2}(t-2), a_{2}^{\prime}(t-1)=a_{2}(t-1)$ при $\tau \geqslant 3, t \geqslant 3$, но $a_{2}^{\prime}(t) \neq \sigma_{\varphi\left(a_{2}^{\prime}\right)}(0)$, то есть $a_{2}^{\prime}(t) \neq \sigma_{\varphi\left(a_{2}\right)}(0)$. Из определения семейства отношений $V_{k}^{\tau}$ следует, что набор $\left(a_{1}, a_{2}^{\prime}\right)$ не принадлежит $R$. Вместе с тем, как нетрудно видеть, существует $S$-функция $f(x)$ такая, что $f\left(a_{2}\right)=a_{2}^{\prime}$ и $f(x) \notin S(U(R))$ и $S\left(P_{k}^{\tau}(1)\right) \not \subset S(U(R))$.

Предложение 6. Пусть $k \in\{2,3,4\}$. Пусть набор $\left(a_{1}, a_{2}, a_{3}, a_{4}\right)$ принадлежит множеству $E_{k} \times E_{k} \times E_{k} \times E_{k}$. Пусть $G\left(E_{k}, \oplus\right)-$ произвольная элементарная абелева 2-группа, если $k=2, k=4$, и 3-группа, если $k=3$. Равенство

$$
a_{1} \oplus a_{2}=a_{3} \oplus a_{4}
$$

справедливо тогда и только тогда, когда выполняются следующие условия.

(a) Пусть $a_{1}=a_{2}$. Тогда, если $k=2, k=4$, то $a_{3}=a_{4}$. если $k=3$, то либо $a_{3}=a_{4}=a_{2}=a_{1}$, либо $a_{3} \neq a_{4}, a_{3} \neq a_{1}, a_{4} \neq a_{1}$.

(б) Пусть $a_{1} \neq a_{2}$. Тогда, если $k=2$, то $a_{3} \neq a_{4}$, если $k=3$, то $a_{3}=a_{1}, a_{4}=a_{2}$ или $a_{3}=a_{2}, a_{4}=a_{1}$, либо $a_{3}=a_{4}, a_{1} \neq a_{3}, a_{2} \neq a_{3}$, если $k=4$, то либо $a_{3}=a_{1}$, $a_{4}=a_{2}$ или $a_{3}=a_{2}, a_{4}=a_{1}$, либо $a_{3} \neq a_{4}, a_{1} \neq a_{3}, a_{2} \neq a_{3}, a_{2} \neq a_{4}$.

Доказательство. Пусть число $b$ из $E_{k}-$ ноль группы $G\left(E_{k}, \oplus\right)$. Если $k=2$, то справедливость предложения 6 очевидна.

Пусть $k=3$. Из определения элементарной абелевой 3-группы $G\left(E_{3}, \oplus\right)$ следует, что для любого $a \in E_{3}$ такого, что $a \oplus a \oplus a=b$, если $a \neq b$, то $a \oplus a \neq b, a \oplus a \neq a$, если $a$ и $a^{\prime}$ из $E_{3}$ таковы, что $a \neq a^{\prime}, a \neq b, a^{\prime} \neq b$, то $a \oplus a^{\prime}=b$.

Пусть $k=4$. Из определения элементарной абелевой 2-группы $G\left(E_{4}, \oplus\right)$ следует, что для любого $a \in E_{4}$ такого, что $a \oplus a=b$, если $a$ и $a^{\prime}$ из $E_{4}$ таковы, что $a \neq a^{\prime}, a \neq b$, $a^{\prime} \neq b$, то $a \oplus a^{\prime} \neq a, a \oplus a^{\prime} \neq a^{\prime}, a \oplus a^{\prime} \neq b$.

Используя эти свойства групп $G\left(E_{3}, \oplus\right)$ и $G\left(E_{4}, \oplus\right)$ легко убедиться в том, что для любого набора $\left(a_{1}, a_{2}, a_{3}, a_{4}\right)$, удовлетворяющего свойствам (а) и (б) при $k=3$ и $k=4$ равенство (1) выполнено; и наоборот, для любого набора, для которого имеет место равенство (1), справедливы свойства (а) и (б).

Предложение 7. Пусть $k \in\{2,3,4\}$. Пусть $G\left(E_{k}, \oplus\right), G\left(E_{k},+\right)$ - элементарньле абелевы 2-группь, если $k=2, k=4$, и 3-группьл, если $k=3$; при этом будем считать, что операция + в группах $G\left(E_{2},+\right), G\left(E_{3},+\right), G\left(E_{4},+\right)$ задается так же, как и при определении семейств отношений $L_{2}(2, \tau), L_{3}(3, \tau), L_{4}(4, \tau)$. Пусть наборы $\left(a_{1}, a_{2}, a_{3}, a_{4}\right) u$ $\left(a_{1}^{\prime}, a_{2}^{\prime}, a_{3}^{\prime}, a_{4}^{\prime}\right)$ из $E_{k} \times E_{k} \times E_{k} \times E_{k}$ таковы, что для некоторых определенных на $E_{k}$ подстановок (перестановок) $\sigma_{1} и \sigma_{2}$

$$
\begin{gathered}
a_{1}^{\prime}=\sigma_{1}\left(a_{1}\right), \quad a_{2}^{\prime}=\sigma_{1}\left(a_{2}\right), \quad a_{3}^{\prime}=\sigma_{1}\left(a_{3}\right), \quad a_{4}^{\prime}=\sigma_{1}\left(a_{4}\right), \\
\sigma_{2}\left(a_{1}\right) \oplus \sigma_{2}\left(a_{2}\right)=\sigma_{2}\left(a_{3}\right) \oplus \sigma_{2}\left(a_{4}\right) .
\end{gathered}
$$

Тогда для любой подстановки (перестановки) $\sigma$, определенной на $E_{k}$,

$$
\sigma\left(a_{1}^{\prime}\right) \oplus \sigma\left(a_{2}^{\prime}\right)=\sigma\left(a_{3}^{\prime}\right) \oplus \sigma\left(a_{4}^{\prime}\right) .
$$


Кроме того, имеют место равенства

$$
a_{1}+a_{2}=a_{3}+a_{4}, \quad a_{1}^{\prime}+a_{2}^{\prime}=a_{3}^{\prime}+a_{4}^{\prime} .
$$

Доказательство. Заметим, что если для набора $\left(a_{1}, a_{2}, a_{3}, a_{4}\right)$ справедливо одно из свойств (а) или (б) предложения 6, то то же свойство справедливо и для наборов $\left(a_{1}^{\prime}, a_{2}^{\prime}, a_{3}^{\prime}, a_{4}^{\prime}\right),\left(\sigma\left(a_{1}^{\prime}\right), \sigma\left(a_{2}^{\prime}\right), \sigma\left(a_{3}^{\prime}\right), \sigma\left(a_{4}^{\prime}\right)\right)$. Поэтому $\sigma\left(a_{1}^{\prime}\right) \oplus \sigma\left(a_{2}^{\prime}\right)=\sigma\left(a_{3}^{\prime}\right) \oplus \sigma\left(a_{4}^{\prime}\right)$. Вместе с тем, согласно предложению 6, элементарные абелевы 2-группы и 3-группы могут выбираться произвольно. Отсюда следует, что $a_{1}+a_{2}=a_{3}+a_{4}, a_{1}^{\prime}+a_{2}^{\prime}=a_{3}^{\prime}+a_{4}^{\prime}$.

Предложение 8. Пусть $k \in\{2,3,4\}, R-$ произвольное отношение из семейства $L(k, \tau)$. Тогда $S\left(P_{k}^{\tau}(1)\right) \subset S(U(R))$, причем $R \in L_{2}(2, \tau)$ при $k=2, R \in L_{3}(3, \tau)$ при $k=3$, $R \in L_{4}(4, \tau)$ при $k=4$.

Доказательство. Пусть $R \subset E_{k}^{t} \times E_{k}^{t} \times E_{k}^{t} \times E_{k}^{t}$ для некоторых $t, 1 \leqslant t \leqslant \tau, \varphi \in \Phi_{t}$. Пусть набор $\left(a_{1}, a_{2}, a_{3}, a_{4}\right)$ принадлежит $R$. Это означает, что

$$
\sigma_{\varphi\left(a_{1}\right)}\left(a_{1}(t)\right) \oplus \sigma_{\varphi\left(a_{1}\right)}\left(a_{2}(t)\right)=\sigma_{\varphi\left(a_{1}\right)}\left(a_{3}(t)\right) \oplus \sigma_{\varphi\left(a_{1}\right)}\left(a_{4}(t)\right) .
$$

Пусть $f(x)$ - произвольная $S$-функция из $P_{k}^{\tau}$ и $f\left(a_{1}\right)=a_{1}^{\prime}, f\left(a_{2}\right)=a_{2}^{\prime}, f\left(a_{3}\right)=a_{3}^{\prime}$, $f\left(a_{4}\right)=a_{4}^{\prime}$. Так как $f(x)-S$-функция, существует, как не трудно видеть, определенная на $E_{k}$ подстановка (перестановка) $\sigma$ такая, что $a_{1}^{\prime}(t)=\sigma\left(a_{1}(t)\right), a_{2}^{\prime}(t)=\sigma\left(a_{2}(t)\right)$, $a_{3}^{\prime}(t)=\sigma\left(a_{3}(t)\right), a_{4}^{\prime}(t)=\sigma\left(a_{4}(t)\right)$. Из предложения 7 следует, что тогда

$$
\sigma_{\varphi\left(a_{1}^{\prime}\right)}\left(a_{1}^{\prime}(t)\right) \oplus \sigma_{\varphi\left(a_{1}^{\prime}\right)}\left(a_{2}^{\prime}(t)\right)=\sigma_{\varphi\left(a_{1}^{\prime}\right)}\left(a_{3}^{\prime}(t)\right) \oplus \sigma_{\varphi\left(a_{1}^{\prime}\right)}\left(a_{4}^{\prime}(t)\right) .
$$

Однако это означает, что набор $\left(a_{1}^{\prime}, a_{2}^{\prime}, a_{3}^{\prime}, a_{4}^{\prime}\right)$ также принадлежит $R$. Таким образом, $f(x) \in S(U(R))$ и $S\left(P_{k}^{\tau}(1)\right) \subset S(U(R))$. Вместе с тем, $a_{1}(t)+a_{2}(t)=a_{3}(t)+a_{4}(t)$ и $a_{1}^{\prime}(t)+a_{2}^{\prime}(t)=a_{3}^{\prime}(t)+a_{4}^{\prime}(t)$. Поэтому $R \in L_{2}(2, \tau)$, если $k=2, R \in L_{3}(3, \tau)$, если $k=3$, $R \in L_{4}(4, \tau)$, если $k=4$.

Предложение 9. Пусть $k \geqslant 5, \tau \geqslant 1, R$ - произвольное отношение из семейства $L(k, \tau)$. Тогда $S\left(P_{k}^{\tau}(1)\right) \not \subset S(U(R))$.

Доказательство. Пусть $R \subset E_{k}^{t} \times E_{k}^{t} \times E_{k}^{t} \times E_{k}^{t}$ для некоторых $t, 1 \leqslant t \leqslant \tau$, и $\varphi \in \Phi_{t}$. Нетрудно видеть, что в $R$ существует набор $\left(a_{1}, a_{2}, a_{3}, a_{4}\right)$ такой, что $a_{1}(t), a_{2}(t), a_{3}(t)$, $a_{4}(t)$ попарно различны и

$$
\sigma_{\varphi\left(a_{1}\right)}\left(a_{1}(t)\right) \oplus \sigma_{\varphi\left(a_{1}\right)}\left(a_{2}(t)\right)=\sigma_{\varphi\left(a_{1}\right)}\left(a_{3}(t)\right) \oplus \sigma_{\varphi\left(a_{1}\right)}\left(a_{4}(t)\right) .
$$

Так как $k \geqslant 5$, в $E_{k}$ существует $a^{\prime}$ такое, что $a^{\prime} \neq a_{4}(t)$, и следовательно,

$$
\left.\sigma_{\varphi\left(a_{1}\right)}\left(a_{1}(t)\right) \oplus \sigma_{\varphi\left(a_{1}\right)}\left(a_{2}(t)\right) \neq \sigma_{\varphi\left(a_{1}\right)}\left(a_{3}(t)\right) \oplus \sigma_{\varphi\left(a_{1}\right)}\left(a^{\prime}\right)\right) .
$$

Рассмотрим набор $\left(a_{1}, a_{2}, a_{3}, a_{4}^{\prime}\right)$ из $E_{k}^{t} \times E_{k}^{t} \times E_{k}^{t} \times E_{k}^{t}$ такой, что $a_{4}^{\prime}(t)=a^{\prime}$, и если $t \geqslant 2, \tau \geqslant 2$, то $a_{4}^{\prime}(1)=a_{4}(1), \ldots, a_{4}^{\prime}(t-1)=a_{4}(t-1)$. Легко убедиться в том, что в $P_{k}^{\tau}$ существует $S$-функция $f(x)$ такая, что $f\left(a_{1}\right)=a_{1}, f\left(a_{2}\right)=a_{2}, f\left(a_{3}\right)=a_{3}, f\left(a_{4}\right)=a_{4}^{\prime}$. Ho

$$
\sigma_{\varphi\left(a_{1}\right)}\left(a_{1}(t)\right) \oplus \sigma_{\varphi\left(a_{1}\right)}\left(a_{2}(t)\right) \neq \sigma_{\varphi\left(a_{1}\right)}\left(a_{3}(t)\right) \oplus \sigma_{\varphi\left(a_{1}\right)}\left(a_{4}^{\prime}(t)\right) .
$$

Поэтому набор $\left(a_{1}, a_{2}, a_{3}, a_{4}^{\prime}\right)$ не принадлежит отношению $R$. Отсюда следует, что $f(x) \notin S(U(R))$ и $S\left(P_{k}^{\tau}(1)\right) \not \subset S(U(R))$. 
Предложение 10. Пусть $k>5, \tau \geqslant 1, R-$ произвольное отночение из семейства $I_{0}(k, \tau)$. Тогда $S\left(P_{k}^{\tau}(1)\right) \not \subset S(U(R))$.

Доказательство. Из определения семейства отношений $I_{0}(k, \tau)$ следует, что $k$ представимо в виде $k=h^{m}$, где $h \geqslant 5, m>1$. Пусть для некоторых $t, 1 \leqslant t \leqslant \tau$, и $\varphi \in \Phi_{t}$ справедливо включение $R \subset E_{k}^{t} \times E_{k}^{t}$. Пусть набор $\left(a_{1}, a_{2}\right)$ принадлежит $R$. Это означает, что для любого $i, 1 \leqslant i \leqslant m, i$-е компоненты чисел $\sigma_{\varphi\left(a_{1}\right)}\left(a_{1}(t)\right)$ и $\sigma_{\varphi\left(a_{1}\right)}\left(a_{2}(t)\right)$ при разложении их по степеням числа $h$ различны. Так как $m>1$, очевидно, что в $E_{k}$ существуют $\tilde{a}_{1}$ и $\tilde{a}_{2}$ такие, что $\tilde{a}_{1} \neq \tilde{a}_{2}$ и первые компоненты чисел $\tilde{a}_{1}$ и $\tilde{a}_{2}$ при разложении их по степеням числа $h$ совпадают. Вместе с тем, существуют $\tilde{a}_{1}^{\prime}$ и $\tilde{a}_{2}^{\prime}$, принадлежащие $E_{k}$, такие, что $\sigma_{\varphi\left(a_{1}\right)}\left(\tilde{a}_{1}^{\prime}\right)=\tilde{a}_{1}, \sigma_{\varphi\left(a_{1}\right)}\left(\tilde{a}_{2}^{\prime}\right)=\tilde{a}_{2}$. Рассмотрим набор $\left(a_{1}^{\prime}, a_{2}^{\prime}\right)$ из $E_{k}^{t} \times E_{k}^{t}$ такой, что $a_{1}^{\prime}(t)=\tilde{a}_{1}^{\prime}, a_{2}^{\prime}(t)=\tilde{a}_{2}^{\prime}$ и $a_{1}^{\prime}(1)=a_{2}^{\prime}(1)=a_{1}(1), \ldots, a_{1}^{\prime}(t-1)=a_{2}^{\prime}(t-1)=a_{1}(t-1)$ при $\tau \geqslant 2, t \geqslant 2$. Очевидно, набор $\left(a_{1}^{\prime}, a_{2}^{\prime}\right)$ не принадлежит $R$. Однако в $P_{k}^{\tau}$ существует $S$-функция $f(x)$ такая, что $f\left(a_{1}\right)=a_{1}^{\prime}, f\left(a_{2}\right)=a_{2}^{\prime}$. Ясно, что $S$-функция $f(x) \notin S(U(R))$. Поэтому $S\left(P_{k}^{\tau}(1)\right) \not \subset S(U(R))$.

Предложение 11. Пусть $k \geqslant 5, \tau \geqslant 1, R-$ произвольное отночение из семейства $I_{1}(k, \tau)$. Тогда $S\left(P_{k}^{\tau}(1)\right) \subset S(U(R))$.

Доказательство. Пусть для некоторого $t, 1 \leqslant t \leqslant \tau, R \subset E_{k}^{t} \times E_{k}^{t}$. Пусть $\left(a_{1}, a_{2}\right)-$ произвольный набор из $R$ и $f(x)$ - произвольная $S$-функция из $P_{k}^{\tau}$. Пусть $f\left(a_{1}\right)=a_{1}^{\prime}$, $f\left(a_{2}\right)=a_{2}^{\prime}$. Из определения семейства отношений $I_{1}(k, \tau)$ следует, что $a_{1}(t) \neq a_{2}(t)$, и если $\tau \geqslant 2, t \geqslant 2$, то $a_{2}(1)=a_{1}(1), \ldots, a_{2}(t-1)=a_{1}(t-1)$. Очевидно, тому же свойству будет удовлетворять и набор $\left(a_{1}^{\prime}, a_{2}^{\prime}\right)$. Таким образом, набор $\left(a_{1}^{\prime}, a_{2}^{\prime}\right)$ также принадлежит $R$. Поэтому $f(x) \in S(U(R))$ и $S\left(P_{k}^{\tau}(1)\right) \subset S(U(R))$.

Нетрудно видеть, что из справедливости предложений 1-11 следует справедливость теоремы 6.

Пусть $k \geqslant 2, \tau \geqslant 1, W_{1}(k, \tau)$ - объединение семейств $N(2, \tau), L_{2}(2, \tau), L_{3}(3, \tau)$, $L_{4}(4, \tau), I_{1}(k, \tau)$.

Непосредственным следствием теорем 5 и 6 является следующее утверждение.

Теорема 7. Пусть $k \geqslant 2, \tau \geqslant 1$. Произвольное $S$-множество $\mathfrak{M}$, принадлежашее совокупности $S$-множеств $M_{k}^{\tau}$, является полным в $P_{k}^{\tau}$ тогда и только тогда, когда $\mathfrak{M}$ не сохраняет ни одного отночения из $W_{1}(k, \tau)$.

Определение 9. Пусть $k \geqslant 2, n \geqslant 2$. $S$-функция $f\left(x_{1}, \ldots, x_{n}\right)$ из $P_{k}^{1}$ (или из $P_{k}$ ) называется существенной, если она существенно зависит не менее чем от двух переменных.

Предложение 12. Пусть $k \geqslant 3, n \geqslant 2, f\left(x_{1}, \ldots, x_{n}\right)-$ произвольная существенная $S$-функция из $P_{k}$. Сущесттвуют наборы $A_{1}=\left(a_{1}^{1}, \ldots, a_{n}^{1}\right), A_{2}=\left(a_{1}^{2}, \ldots, a_{n}^{2}\right)$ и $S$-функции $f_{1}(x), \ldots, f_{n}(x)$ такие, что $a_{1}^{1} \neq a_{1}^{2}, \ldots, a_{n}^{1} \neq a_{n}^{2} u$

$$
f\left(f_{1}\left(a_{1}^{1}\right), \ldots, f_{n}\left(a_{n}^{1}\right)\right)=f\left(f_{1}\left(a_{1}^{2}\right), \ldots, f_{n}\left(a_{n}^{2}\right)\right) .
$$

Доказательство. Не ограничивая общности, будем считать, что $S$-функция $f\left(x_{1}, \ldots, x_{n}\right)$ существенно зависит от переменных $x_{1}, x_{2}$. Тогда, согласно известной лемме С. В. Яблонского [4], существуют 3 набора $\left(a_{1}, a_{2}, a_{3}, \ldots, a_{n}\right),\left(a_{1}, \tilde{a}_{2}, \tilde{a}_{3}, \ldots, \tilde{a}_{n}\right),\left(\tilde{a}_{1}, \tilde{a}_{2}, \tilde{a}_{3}, \ldots, \tilde{a}_{n}\right)$ такие, что $a_{1} \neq \tilde{a}_{1}, a_{2} \neq \tilde{a}_{2}$ и значения функции $f\left(x_{1}, \ldots, x_{n}\right)$ на этих наборах попарно различны. Пусть $S$-функции $f_{1}(x), \ldots, f_{n}(x)$ и числа $b_{3}, \ldots, b_{n}$ из $E_{2}$ таковы, что $f_{1}(0)=a_{1}, f_{2}(0)=a_{2}, \ldots, f_{n}(0)=a_{n}, f_{1}(1)=\tilde{a}_{1}, f_{2}(1)=\tilde{a}_{2}$ и для любого $i$, 
$3 \leqslant i \leqslant n, b_{i}=0$, если $\tilde{a}_{i}=a_{i} ; b_{i}=1$ и $f_{i}(1)=\tilde{a}_{i}$, если $\tilde{a}_{i} \neq a_{i}$. Рассмотрим функцию $\tilde{f}\left(x_{1}, \ldots, x_{n}\right)=f\left(f_{1}\left(x_{1}\right), \ldots, f_{n}\left(x_{n}\right)\right)$. Нетрудно видеть, что $\tilde{f}\left(x_{1}, \ldots, x_{n}\right)$ является $S$-функцией, причем на наборах $(0, \ldots, 0),\left(0,1, b_{3}, \ldots, b_{n}\right),\left(1,1, b_{3}, \ldots, b_{n}\right)$ эта функция принимает три разных значения.

Пусть существуют $a_{1}^{1}, a_{1}^{2} \in E_{k}$ такие, что $a_{1}^{1} \neq a_{1}^{2}$, но $\tilde{f}\left(a_{1}^{1}, \ldots, a_{1}^{1}\right)=\tilde{f}\left(a_{1}^{2}, \ldots, a_{2}^{2}\right)$, где $\tilde{f}-$ функция от $n$ переменных. Очевидно, что тогда в качестве наборов $A_{1}$ и $A_{2}$ можно взять наборы $\left(a_{1}^{1}, \ldots, a_{1}^{1}\right)$ и $\left(a_{1}^{2}, \ldots, a_{1}^{2}\right)$. Пусть для любых $a$ и $a^{\prime}$ из $E_{k}$ таких, что $a \neq a^{\prime}, \tilde{f}(a, \ldots, a) \neq \tilde{f}\left(a^{\prime}, \ldots, a^{\prime}\right)$. Так как значения $\tilde{f}(0,0,0, \ldots, 0), \tilde{f}\left(0,1, b_{3}, \ldots, b_{n}\right)$, $\tilde{f}\left(1,1, b_{3}, \ldots, b_{n}\right)$ попарно различны, как легко видеть, найдется такое $a_{1}^{2} \in\{2, \ldots, k-1\}$, что либо $\tilde{f}\left(0,1, b_{3}, \ldots, b_{n}\right)=\tilde{f}\left(a_{1}^{2}, \ldots, a_{1}^{2}\right)$, либо $\tilde{f}\left(1,1, b_{3}, \ldots, b_{n}\right)=\tilde{f}\left(a_{1}^{2}, \ldots, a_{1}^{2}\right)$. Отсюда следует, что в этом случае в качестве набора $A_{2}$ можно взять набор $\left(a_{1}^{2}, \ldots, a_{1}^{2}\right)$, а в качестве $A_{1}$ - либо набор $\left(0,1, b_{3}, \ldots, b_{n}\right)$, либо набор $\left(1,1, b_{3}, \ldots, b_{n}\right)$.

Предложение 13. Пусть $k \geqslant 5$, $\mathfrak{M}-$ произвольное $S$-множество из $P_{k}^{1}$. Пусть $R-$ единственное отношение, принадлежащее семейству $I_{1}(k, \tau)$. Тогда

(а) если М состоит только из $S$-функций, которые не являются существенными, то $\mathfrak{M} \subseteq S(U(R))$

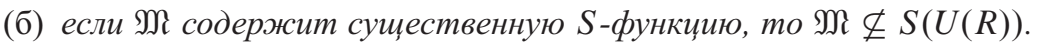

Заметим, что никакая функция из $S\left(P_{k}^{1}\right)$ не является существенной.

С задачей о полноте $S$-множеств в $P_{k}^{\tau}$ тесно связана задача об $A$-полноте систем, состоящих из $S$-ограниченно-детерминированных функций. Пусть $k \geqslant 2$ и $P_{b d}^{k}-$ множество всех ограниченно-детерминированных функций (о.-д. функций), определенных на наборах бесконечных последовательностей, составленных из букв алфавита $E_{k}$. Учитывая детерминированность функций из $P_{b d}^{k}$, можно, очевидно, полагать, что для всякого $t \geqslant 1$ эти функции определены также на всех наборах слов длины $t$, принадлежащих множеству $E_{k}^{t}$. Будем считать, что на множестве $P_{b d}^{k}$ заданы операции суперпозиции и обратной связи. Известно, однако, что при изучении задачи об $A$-полноте операция “обратная связь” оказывается несущественной и выразима через операцию суперпозиции [2]. Так же как и при исследовании задачи о полноте в $P_{k}^{\tau}$, введем понятия замыкания, замкнутого множества и т. п.

Определение 10. Пусть $k \geqslant 2, \tau \geqslant 1$. Ограниченно-детерминированную функцию $g\left(x_{1}, \ldots, x_{n}\right)$ и функцию $f\left(x_{1}, \ldots, x_{n}\right)$ из $P_{k}^{\tau}$ назовем $\tau$-эквивалентными, если $g\left(a_{1}, \ldots, a_{n}\right)=f\left(a_{1}, \ldots, a_{n}\right)$ для любого набора $\left(a_{1}, \ldots, a_{n}\right)$ слов из $E_{k}^{\tau}$.

Определение 11. Пусть $k \geqslant 2, \tau \geqslant 1, \mathfrak{N} \subseteq P_{b d}^{k}, \mathfrak{M} \subseteq P_{k}^{\tau}$. Множества $\mathfrak{N}$ и $\mathfrak{M}$ назовем $\tau$-эквивалентными, если для любой ограниченно-детерминированной функции $g$ из $\mathfrak{N}$ в $\mathfrak{M}$ существует $\tau$-эквивалентная ей ограниченно-детерминированная функция $f$ и наоборот: для любой функции $f^{\prime}$ из $\mathfrak{M}$ в $\mathfrak{N}$ существует ограниченно-детерминированная функция $g^{\prime}$, которая $\tau$-эквивалентна функции $f^{\prime}$.

Заметим, что для всякого $\tau \geqslant 1$ любое множество ограниченно-детерминированных функций $\mathfrak{N} \tau$-эквивалентно некоторому множеству $\mathfrak{M} \subseteq P_{k}^{\tau}$; при этом, если $\mathfrak{M}$ сохраняет некоторое отношение $R$, то и $\Re$ сохраняет это отношение.

Определение 12. Пусть $k \geqslant 2, \mathfrak{N} \subseteq P_{b d}^{k}$. Множество $\mathfrak{N}$ называется $A$-полным, если для любого $\tau \geqslant 1$ замыкание множества $\mathfrak{N} \tau$-эквивалентно множеству $P_{k}^{\tau}$. 
Известно [2], что в общем случае не существует алгоритма для распознавания $A$-полноты конечных множеств из $P_{b d}^{k}$. С этой точки зрения представляет интерес поиск такого алгоритма для систем ограниченно-детерминированных функций, обладающих некоторыми наперед заданными свойствами.

Определение 13. Пусть $k \geqslant 2$. Пусть $g\left(x_{1}, \ldots, x_{n}\right) \in P_{b d}^{k}$. Ограниченно-детерминированную функцию $g\left(x_{1}, \ldots, x_{n}\right)$ назовем $S$-ограниченно-детерминированной функцией, если в каждом ее состоянии реализуется функция $k$-значной логики, не выпускающая ни одного значения из множества $E_{k}$.

Определение 14. Пусть $k \geqslant 2, \mathfrak{N} \subset P_{b d}^{k}$. Множество ограниченно-детерминированных функций $\mathfrak{N}$ назовем $S$-множеством, если любая ограниченно-детерминированная функция из $\mathfrak{\Re}$ является $S$-ограниченно-детерминированной функцией.

Пусть $k \geqslant 2, \mathfrak{N} \subset P_{b d}^{k}$. Через $S(\mathfrak{N})$ обозначим подмножество $\mathfrak{N}$, состоящее из всех

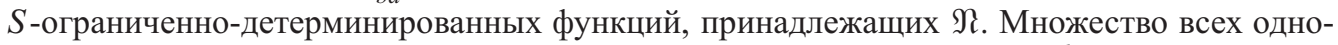
местных $S$-ограниченно-детерминированных функций обозначим $S\left(P_{b d}^{k}(1)\right)$.

Пусть $M_{b d}^{k}-$ совокупность всех $S$-множеств ограниченно-детерминированных функций $\mathfrak{\Re}$ таких, что $S\left(P_{b d}^{k}(1)\right) \subseteq \Re$ и $\backslash S\left(P_{b d}^{k(1)}\right)<\infty$.

Возникает вопрос, при каких условиях произвольное $S$-множество из $M_{b d}^{k}$ является $A$-полным и существует ли алгоритм для распознавания $A$-полноты систем, принадлежащих совокупности $M_{b d}^{k}$.

Непосредственным следствием теоремы 7 является следующее утверждение.

Теорема 8. Пусть $k \geqslant 2$. Произвольное множество ограниченно-детерминированных функций $\mathfrak{\Re , ~ п р и н а д л е ж а щ е е ~ с о в о к у п н о с т и ~} S$-множеств $M_{b d}^{k}$, является $A$-полным тогда и только тогда, когда $\mathfrak{N}$ не сохраняет ни одного отношения из $W_{1}(k, \tau)$ для любого $\tau \geqslant 1$.

Доказательство. Пусть $\mathfrak{\Re}$ является $A$-полным. Это означает, что для любого $\tau \geqslant 1$ замыкание $\mathfrak{\Re} \tau$-эквивалентно $P_{k}^{\tau}$ и, таким образом, не сохраняет ни одного отношения из $W_{1}(k, \tau)$.

Пусть множество $\mathfrak{N}$ не является $A$-полным. Тогда существует $\tau \geqslant 1$ такое, что замыкание $\mathfrak{N}$ не является $\tau$-эквивалентным $P_{k}^{\tau}$. Вместе с тем, $S$-множество ограниченнодетерминированных функций $\mathfrak{\Re} \tau$-эквивалентно некоторому $S$-множеству $\mathfrak{M}$, принадлежащему совокупности множеств $M_{k}^{\tau}$ и не являющемуся полным в $P_{k}^{\tau}$. Но тогда множество $\mathfrak{M}$, и следовательно, множество $\mathfrak{\Re}$ согласно теореме 7 сохраняет некоторое отношение, принадлежащее $W_{1}(k, \tau)$.

Пусть $k \geqslant 2, f\left(x_{1}, \ldots, x_{n}\right)$ - произвольная ограниченно-детерминированная функция из $P_{b d}^{k}$. Через $Q_{f}, q_{0}^{f}, \varphi_{f}\left(q, x_{1}, \ldots, x_{n}\right)$ обозначим соответственно множество состояний, начальное состояние и функцию переходов ограниченно-детерминированной функции $f\left(x_{1}, \ldots, x_{n}\right)$, причем функция $\varphi_{f}\left(q, x_{1}, \ldots, x_{n}\right)$ определена на множестве всех наборов слов одной и той же длины, составленных из букв алфавита $E_{k}$.

Определение 15. Пусть $k \geqslant 2, t \geqslant 1, f\left(x_{1}, \ldots, x_{n}\right) \in P_{b d}^{k}$. Состояние $q \in Q_{f}$ ограниченно-детерминированной функции $f\left(x_{1}, \ldots, x_{n}\right)$ называется $t$-достижимым, если существует набор слов $\left(a_{1}, \ldots, a_{n}\right)$ из $E_{k}^{t}, \ldots, E_{k}^{t}$ (длины $n$ ) такой, что

$$
\varphi_{f}\left(q_{0}^{f}, a_{1}, \ldots, a_{n}\right)=q .
$$

Будем считать при этом, что 0-достижимым состоянием ограниченно-детерминированной функции $f\left(x_{1}, \ldots, x_{n}\right)$ является ее начальное состояние. 
С использованием понятия $t$-достижимости дадим несколько иную формулировку теоремы 8.

Теорема 9. Пусть $k \geq 2, \mathfrak{N} \in M_{b d}^{k}, \mathfrak{N} \backslash S\left(P_{b d}^{k(1)}\right)=\left\{f_{1}\left(x_{1}, \ldots, x_{n_{1}}\right), \ldots, f_{m}\left(x_{1}, \ldots, x_{n_{m}}\right)\right\}$. $S$-множество ограниченно-детерминированных функщий $\mathfrak{\Re}$ является $A$-полным в $P_{b d}^{k}$ тогда и только тогда, когда для любого $t \geqslant 0$ имеют место следующие утверждения.

(1) Если $k=2$, то сущуествуют $l$ и $l^{\prime}, 1 \leqslant l, l^{\prime} \leqslant m, u$-достижимые состояния $q \in Q_{f_{l}}, q^{\prime} \in Q_{f_{l}^{\prime}}$ такие, что в состоянии q реализуется нелинейная, а в состоянии $q^{\prime}$ несамодвойственная $S$-функиии алгебры логики (предложения 4 и 8);

(2) Если $k=3, k=4$, то сущуествуют $l, 1 \leqslant l \leqslant m$, и $t$-достижимое состояние $q \in Q_{f_{l}}$ такие, что в состоянии q реализуется $S$-функция $k$-значной логики (или $S$-функция из $\left.P_{k}^{1}\right)$, не сохраняющая единственного отношения, принадлежащего семействам $L_{3}(3,1)\left(L_{4}(4,1)\right.$ соответственно) (предложение 8);

(3) Если $k \geqslant 5$, то существуют $l, 1 \leqslant l \leqslant m$, и t-достижимое состояние $q \in Q_{f_{l}}$ такие, что в состоянии q реализуется существенная $S$-функция $k$-значной логики (или сущеественная $S$-функция из $P_{k}^{1}$ ) (предложения 13 и 14).

Заметим, что для любых $k \geqslant 2, \tau \geqslant 1$ теорема, аналогичная теореме 9, справедлива и при исследовании задачи о полноте в $P_{k}^{\tau} S$-множеств, принадлежащих совокупности $M_{k}^{\tau}$. Для этого в формулировке теоремы 9 достаточно рассматривать $t$ такие, которые не превосходят числа $\tau-1$.

Теорема 10. Пусть $k \geqslant 2$. Существует алгоритм для распознавания А-полнотьл произвольных множеств ограниченно-детерминированных функций, принадлежащих совокупности $S$-множеств $M_{b d}^{k}$.

Очевидно, справедливо следующее утверждение.

Предложение 14. Пусть $k \geqslant 2$. Сущуествует алгоритм, для любой $S$-функиии из $P_{k}$ (или из $\left.P_{k}^{1}\right)$ устанавливающий, является ли эта функция линейной или самодвойственной при $k=2$, сохраняет ли эта функичи отношения из семейств $L_{3}(3,1), L_{4}(4,1)$ при $k=3$, $k=4$, является ли эта функичи существенной при $k \geqslant 5$.

Пусть $k \geqslant 2, f-$ произвольная ограниченно-детерминированная функция из $P_{b d}^{k}$. Пусть $t \geqslant 0$. Через $Q_{f}(t)$ обозначим подмножество $Q_{f}$, содержащее все $t$-достижимые состояния ограниченно детерминированной функции $f$.

Предложение 15. Пусть $k \geqslant 2$, $\mathfrak{N}$ - произвольное $S$-множество ограниченно-детерминированных функиий, принадлежащее совокупности $S$-множеств $M_{b d}^{k}$. Пусть $\mathfrak{\Re} \backslash P_{b d}^{k}(1)=\left\{f_{1}, \ldots, f_{n}\right\}$ и $p_{i}-$ число состояний ограниченно-детерминированной функичии $f_{i}$ для $i, 1 \leqslant i \leqslant n$. Пусть $\tau=2^{p_{1}+\ldots+p_{n}} . S$-множество $\mathfrak{\Re}$ является $A$-полным 6 $P_{b d}^{k}$ тогда и только тогда, когда $S$-множество $\mathfrak{M} \subset P_{k}^{\tau}, \tau$-эквивалентное $\mathfrak{N}$, является полнылм в $P_{k}^{\tau}$.

Пусть $Q_{f_{1}}, \ldots, Q_{f_{n}}$ - множества состояний ограниченно-детерминированных функций $f_{1}, \ldots, f_{n}$ соответственно. Для любого $t \geqslant 0$ рассмотрим $Q(t)=\left(Q_{f_{1}}(t), \ldots, Q_{f_{n}}(t)\right)$. Ясно, что если $Q\left(\tau_{1}\right)=Q\left(\tau_{2}\right)$ для некоторых $\tau_{1} \geqslant 0, \tau_{2} \geqslant 0$, то $Q\left(\tau_{1}+t\right)=Q\left(\tau_{2}+t\right)$ для любого $t \geqslant 0$. Вместе с тем, как нетрудно видеть, число различных наборов при разных 
$t$ не превосходит $\tau$. Поэтому, если условия теоремы 9 выполнены для всех $t \leqslant \tau$, то эти условия имеют место и для любого $t \geqslant 0$.

Из справедливости предложений 14, 15 следует справедливость теоремы 10.

Заметим, что аналогичный результат в общем случае, когда рассматриваются произвольные множества (а не только $S$-множества), содержащие все одноместные ограниченно-детерминированные функции, получен в [6]. Однако критерий $A$-полноты таких множеств формулируется значительно сложнее, чем в данной работе.

\section{Список литературы}

1. Буевич В. А., Подколзина М. А., Критерий полноты $S$-множеств детерминированных функций. Матем. вопросы кибернетики (2007) 16, 191-238.

2. Буевич В. А., Об алгоритмической неразрешимости распознавания $A$-полноты для ограниченнодетерминированных функций. Матем. заметки (1972) 6, 687-697.

3. Кудрявцев В. Б., О свойствах $S$-систем функций $k$-значной логики. Elektronische Informationsverarbeitung und Kybernetik (1973) 9, №1-2, 81-105.

4. Яблонский С. В., Функциональные построения в $k$-значных логиках. Труды Математического Института им. В. А. Стеклова (1958) 51, 5-142.

5. Буевич В. А., О $\tau$-полноте систем, содержащих все одноместные детерминированные функции. Матем. вопросы кибернетики (1999) 8, 231-254.

6. Буевич В. А., Клиндукова Т. Э., О существовании алгоритма для распознавания $A$-полноты систем, содержащих все одноместные ограниченно-детерминированные функции. Maтем. вопросы кибернетики (1999) 8, 289-297.

7. Slupecki J., Kryterium pelnosci wielowartosciowych systemów logiki zdan. Comptes Rendus des Séances de la Société des Lettres de Varsovie, Classe III, 32 Année (1939) 102-109.

8. Salomaa A., On basis groups for the set of functions over a finite domain. Ann. Acad. Sci. Finnicae (1963) 338, 1-15.

9. Rosenberg I., Über die funktionale Vollständigkeit in den mehrwertigen Logiken. Rozpr. Česk. Akad. Věd, Řada Mat. Př́r. Věd (1970) 80, №4, 3-93.

Статья поступила 17.09.2008. 